\title{
The Role of 5-Aminolevulinic Acid (5-ALA) and Sleep*
}

\author{
Michael H. Perez ${ }^{1 \#}$, Terry T. Shintani ${ }^{1}$, Beatriz L. Rodriguez ${ }^{2}$, James Davis ${ }^{1}$, Rosanne C. Harrigan ${ }^{1}$ \\ ${ }^{1}$ Department of Complementary and Alternative Medicine, University of Hawaii, John A. Burns School of Medicine, Honolulu, USA; \\ ${ }^{2}$ Department of Geriatric Medicine, University of Hawaii, John A. Burns School of Medicine, Honolulu, USA. \\ Email: "mhperez@hawaii.edu
}

Received August $3^{\text {rd }}, 2013$; revised August $30^{\text {th }}, 2013$; accepted September $20^{\text {th }}, 2013$

Copyright (C 2013 Michael H. Perez et al. This is an open access article distributed under the Creative Commons Attribution License, which permits unrestricted use, distribution, and reproduction in any medium, provided the original work is properly cited.

\begin{abstract}
Objective: To determine if there is a relationship between the administration of the dietary supplement containing 5-Aminolevulinic Acid (5-ALA) and sleep. Methods: A double-blind, randomized parallel-group study was conducted. It was a 4-month study of 40 participants between the ages of 40 and 70 . Males and females were recruited equally. There were 20 in each group who had existing sleep disorders. The tool used to measure participant sleep improvement included the Pittsburgh Insomnia Rating Scale-20 Question (PIRS-20). Improved sleep is reflected when the total PIRS-20 score is lower. Results: Improvement in sleep in the group taking $50 \mathrm{mg}$ 5-ALA, compared to controls, was significant. The mean change, from baseline through week 6 , was -5.67 units less on the sleep scale than the control group with a $\mathrm{p}$ value of 0.001 . The mean change from week 6 to week 10 when the participant was no longer taking the supplement was 4.55 units higher than the control group with a $p$ value of 0.062 , which is of borderline significance. Conclusions: There is a relationship between the administration of dietary supplements containing 5-ALA and sleep. The results of this study suggest that 5-ALA is associated with improved sleep. The mechanism for sleep improvement needs to be explored. Further research is warranted.
\end{abstract}

Keywords: Insomnia; Heme; Energy; Metabolism; 5-Aminolevulinic Acid; Sleep

\section{Introduction}

According to the United States Centers for Disease Control, "Insufficient sleep is associated with a number of chronic diseases and conditions - such as diabetes, cardiovascular disease, obesity, and depression-which threaten our nation's health" [1]. However, most sleep remedies can cause other problems such as drowsiness that can increase the risk of motor vehicle and machinery related accidents. They can even contribute to depression and sleep disorders. Thus, there is great value in finding a natural sleep-aid that does not cause drowsiness or other negative side effects.

5-Aminolevulinic Acid (5-ALA) is a naturally occurring substance and is the basic building block of both heme and chlorophyll. During a previous trial of the oral administration of 5-ALA, some preliminary reports from

\footnotetext{
${ }^{*}$ Conflicts of Interests: The authors declare that they have no conflicts of interest.

Financial Support: The Supplement Sleep and Mood Study's were funded by SBI Pharmaceuticals Co., Ltd. The authors were partially supported by grants from the National Institute on Minority Health and Health Disparities U54MD007584 and G12MD007601 from the National Institutes of Health.

\#Corresponding author.
}

participants suggested that 5-ALA might improve sleep. The purpose of this investigation is to determine if a relationship exists between the administration of dietary supplements containing 5-ALA and sleep.

\section{Background}

5-Aminolevulinic Acid is a natural non-alpha amino acid. 5-ALA is a delta amino acid and is not a component of protein. It is found in many common foods and fermented products such as green pepper, bananas, baker's yeast, vinegar, etc. (see Table 1).

5-ALA is synthesized in the mitochondria. It is a building block of protoporphyrin and a precursor of both chlorophyll and heme. 5-ALA has been associated with the origin of life.

The structure of 5-ALA is described in Figure 1. In the prophyrin synthesis pathway, 5-ALA is the first compound. In mammals, this pathway leads to the synthesis of heme and in plants, where chlorophyll is synthesized. Heme plays a role in oxygen transport and cellular energy production [2]. Cellular energy generation uses membrane-localized heme-based electron transfer chains for adenosine-5'-triphosphate (ATP) synthesis 
Table 1. Common foods that contain 5-ALA.

\begin{tabular}{ccc}
\hline FOOD & 5-ALA content & \\
\hline Spinach & 0.18 & $\mathrm{mg} / \mathrm{kg}$ \\
Green pepper & 0.23 & $\mathrm{mg} / \mathrm{kg}$ \\
Tomato & 0.13 & $\mathrm{mg} / \mathrm{kg}$ \\
Shitake mushroom & 0.60 & $\mathrm{mg} / \mathrm{kg}$ \\
Potato & 0.12 & $\mathrm{mg} / \mathrm{kg}$ \\
Banana & 0.40 & $\mathrm{mg} / \mathrm{kg}$ \\
Squid & 0.50 & $\mathrm{mg} / \mathrm{kg}$ \\
Octopus & 1.00 & $\mathrm{mg} / \mathrm{kg}$ \\
\hline FERMENTED PRODUCTS & & \\
\hline Shochu lees & 70 & $\mathrm{mg} / \mathrm{kg}$ \\
Sake lees & $9-26$ & $\mathrm{mg} / \mathrm{kg}$ \\
Baker's yeast & 140 & $\mathrm{mg} / \mathrm{kg}$ \\
Wine & $1.4-2.2$ & $\mathrm{mg} / \mathrm{L}$ \\
Vinegar & $0.1-5$ & $\mathrm{mg} / \mathrm{L}$ \\
Sweet sake & $0.4-6$ & $\mathrm{mg} / \mathrm{L}$ \\
Sake for cooking & $0.3-13$ & $\mathrm{mg} / \mathrm{L}$ \\
Sake & $0.9-4.5$ & $\mathrm{mg} / \mathrm{L}$ \\
Soy sauce & 0.3 & $\mathrm{mg} / \mathrm{L}$ \\
\hline
\end{tabular}

5-Aminolevulinic Acid

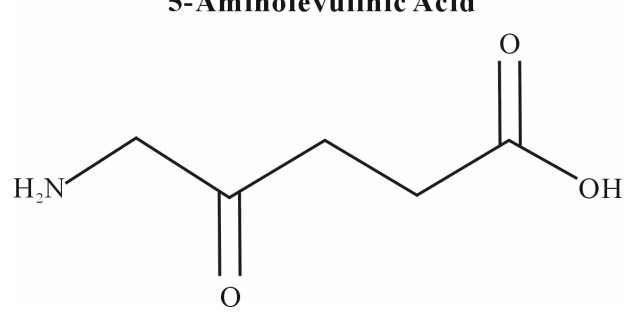

Figure 1. Molecular formula: $\mathrm{C}_{5} \mathrm{H}_{9} \mathrm{NO}_{3}$, average mass: 131.130005 Da, monoisotopic mass: 131.057999 Da [19].

which is used for metabolic energy.

Increased presence of glucose in the cell results in decreased 5-ALA production [3]. At the same time, decreased heme production occurs with aging. This results in decreased heme enzyme activity. A decline in the mitochondrial electron transfer system follows, with decreased basal metabolism. This may result in physical energy decline and possibly depression and sleep disturbance. Understanding the relationship between 5-ALA, heme and energy production might provide evidence that explains why 5-ALA may have a relationship with sleep.

\subsection{Sleep and 5-ALA}

In a previous study conducted to examine the relationship between 5-ALA and pre-diabetes, a questionnaire covering a wide range of measures of health was administered. The study placed participants on the 5-ALA supplement up to $50 \mathrm{mg}$ per day for a period of 12 Weeks.
During the course of this study, some interesting results relating to sleep patterns emerged. Table 2 provides a summary of the survey results pertaining to sleep in some of the participants.

These data support the hypothesis that 5-ALA may be related to improved sleep. The fact that sleep patterns improved while on the $50 \mathrm{mg}$ supplement, and then returned to previous patterns when the supplement was stopped, provide strong rationale for this pilot investigation.

\subsection{Hypothesis Related to Sleep}

There are several possible mechanisms hypothesized for the improvement in sleep. Five-ALA may have an impact on increasing the energy of all cells. In one study involving test mice, researchers found that the regular administration of 5-ALA appeared to raise serotonin levels in the brain [4]. This may explain the improvements in sleep patterns.

Another possible hypothesis is that 5-ALA helps each cell's metabolism, such that its own circadian rhythms are better defined. One may also speculate that 5-ALA may support hormonal regulation, including melatonin production, in the pineal gland-which may result in better sleep and corticosteroid production in the adrenal glands. This may also assist in dealing with stress which may reduce a potential cause of sleep disruption.

\section{Methods}

\subsection{Design}

This was a double-blind, randomized parallel-group comparison study. The intervention group was placed on $50 \mathrm{mg}$ /day of 5-ALA with $57.4 \mathrm{mg}$ of Sodium Ferrous Citrate (SFC), and the comparison group was placed on a placebo (see Table 3). The capsules were administered daily for 6 weeks. Participants were assessed at Baseline, week 3 and week 6 and re-assessed at week 4 when no longer taking the supplement. The study staff was blinded to the intervention group.

\subsection{Sample}

40 participants were randomized to the following 2 study groups: Control Group - 20 participants, and Intervention Group - 20 participants. A table of randomized numbers was used to assign the participants.

\subsection{Procedures}

Recruitment. Advertisements were prepared for the newspaper, TV and radio. Postcards were mass mailed to geographical locations in Hawaii to recruit participants. Flyers and newspaper ads were distributed and local MD's and organizations were contacted. 
Table 2. Results of pilot investigation related to sleep.

\begin{tabular}{|c|c|c|c|}
\hline & $\begin{array}{c}\text { Sleep at } \\
\text { Week } 0 \text { (first day to start 5-ALA) }\end{array}$ & $\begin{array}{c}\text { Sleep at } \\
\text { Week } 12 \text { (12 wks 5-ALA intake) }\end{array}$ & $\begin{array}{c}\text { Sleep at } \\
\text { Week } 16 \text { (4 wks post 5-ALA) }\end{array}$ \\
\hline $\begin{array}{c}\text { Case } \\
\# 4\end{array}$ & 6.5 hours, wakes up several times & 7.25 hours, mostly continuous sleep & $\begin{array}{l}\text { Pattern reverting to waking up several } \\
\text { times at night \& less restful sleep }\end{array}$ \\
\hline $\begin{array}{l}\text { Case } \\
\# 138\end{array}$ & $\begin{array}{l}8.0 \text { hours sleep and does not take a "nap" } \\
\text { during the day, "Moderate" energy }\end{array}$ & $\begin{array}{l}7.5 \text { hours of continuous sleep, able to take a } 10 \text { min. "nap" } \\
\text { during the day, now reports having "Normal" energy }\end{array}$ & Feels "tired”, "no energy”, "sleepy” \\
\hline
\end{tabular}

Table 3. Ingredients of control and intervention supplements.

\begin{tabular}{ccc}
\hline & Control & Intervention \\
\hline 5-ALA Phosphate & $0 \mathrm{mg}$ & $50 \mathrm{mg}$ \\
Sodium Ferrous & $0 \mathrm{mg}$ & $57.4 \mathrm{mg}$ \\
Citrate (SFC) & Alpha starch, & Alpha starch, \\
Other Ingredients & Silicon dioxide & Silicon dioxide \\
\hline
\end{tabular}

Inclusion Criteria. Both males and females were equally recruited. Participants were given questionnaires, and those who were between 40 and 70 years, who selfreported having insomnia or difficulty sleeping, were selected.

Exclusion Criteria. Those at a body weight of $<110$ or $>250 \mathrm{lbs}$ were excluded. Those who were taking any supplements or medications for sleep were also excluded. Those with a history of porphyria were not able to participate, as 5-ALA may cause adverse effects on porphyria patients as it affects porphyrin metabolism. Those with a history of hemochromatosis were excluded, as sodium ferrous citrate (SFC) may cause adverse effects on hemochromatosis patients which have defects in iron metabolism. Those with a history of hepatitis were also excluded, as SFC may cause an allergic reaction in this population. Those with active liver disease and iron sensitivity were also excluded. Women who were pregnant, breastfeeding, and those participating in another clinical study were excluded. Those with ferritin levels elevated above $125 \%$ of normal on screening were excluded.

\subsection{Measures}

\subsubsection{Sleep Study Questionnaire}

Sleep was measured using a lifestyle questionnaire and the Pittsburgh Insomnia Rating Scale-20 Questions (PIRS-20), along with a daily study diary to monitor consistent sleeping or mood patterns.

\subsubsection{Lifestyle Questionnaire}

Leisure-time physical activity and general health was measured using a standardized Lifestyle Questionnaire. The questionnaire covered: Medical history, occurrence of allergy, use of medical drugs and/or health food, drinking, smoking habits, etc.

\subsubsection{PIRS-20}

The PIRS-20 sleep scale is copyrighted by the University of Pittsburgh [5]. In order for the design feature to be a valid sleep scale, questions were used in the original format. The PIRS-20 uses repeated measures to validate answers. A lower score indicates better sleep: Scale-0 (good sleep) to 60 (bad sleep) [5].

\subsubsection{Clinical Examinations}

The examinations recorded participant body weight, Body Mass Index (BMI), seated/resting systolic and diastolic blood pressure and waist circumference.

\subsubsection{Laboratory Tests}

These tests measured baseline Complete Blood Count (CBC) and ferritin levels. Venous blood was obtained and all analyses were conducted using standardized and certified procedures by Diagnostic Laboratory Services (DLS). Samples for selected tests were stored by DLS at appropriate temperatures for assay testing. The same digital scale was used for weighing patients at each site throughout the study. Height was measured in the standing position and measured to within the nearest millimeter without shoes. A stadiometer fitted with a vertical backboard, fixed floorboard, and a movable headboard was utilized. BMI was determined as weight $(\mathrm{kg})$ divided by height squared $\left(\mathrm{m}^{2}\right)$. Waist circumference was measured using a tension-controlled measuring tape while the subject is standing. Abdominal obesity is defined as a $\mathrm{WC} \geq 88 \mathrm{~cm}$ in women and $\geq 102 \mathrm{~cm}$ in men. Blood pressure was measured with a digital sphygmomanometer while patients are in a sitting position. The same arm was used to obtain all readings over the course of the study, unless contraindicated. Sitting systolic and diastolic blood pressures were estimated by averaging 2 replicate measurements obtained 1 to 2 minutes apart. When possible, the same nurse or study coordinator measured waist circumference at each visit to ensure consistency. Results from the lab were reviewed by the medical staff/physician within three business days of receiving the results. There was follow-up activity by contacting the participant and the participant's physician, as appropriate.

\subsection{Study Schedule}

Participants took the supplement from time of enrollment. The measurements occurred at baseline, week 3, week 6 and week 10. At week 10, participants were no longer 
taking the supplement (see Table 4).

\subsection{Efficacy Measures}

A significant difference between sleep and related measures of sleep quality during the examination period between the treatment groups was the major outcome measure. A significant difference in sleep scores between the treatment group and control group at the end of administration is desired and reflective of affirming the hypothesis.

Adverse events data were assessed every two weeks to identify statistically or clinically significant differences between the control group and intervention group, and assessments of whether or not changes to the protocol were needed. If statistically or clinically significant differences in serious adverse events among the placebo and the intervention group were found that indicated that there was a greater risk than benefit for study participants, the study could have ended.

Participants were advised to contact the PI or Co-PI immediately if they had any concerns. If any abnormal findings were identified, and reported the participant would have been removed from the investigation, if warranted.

\subsection{Statistical Analysis}

Variables monitored as part of the evaluation were assessed by comparing the intervention group to the control group. Two-sample t-tests were used to assess statistical significance at baseline and follow-up exams between the control and intervention groups. Baseline data were summarized as means and standard deviations with differences among the randomized groups tested for significance using t-tests and chi-squares. To measure the possible differences in rates of change in sleep scores across follow-up time between the 5-ALA treatment and the control group, an additional analysis was implemented. This consisted of estimating differences in slopes using a linear regression model. Mixed linear models were fit using the proc mixed procedure in SAS 9.2 [6]. The regression models included an indicator variable identifying treatment groups, a variable for weeks of follow-up, and interaction terms between the indicator variables and follow-up time. Results were summarized as the difference in slopes comparing the intervention groups to the control group. Results were also presented graphically to illustrate the estimated differences in slopes for the study groups. All significant tests were two-sided. Differences were considered significant if the $p$ values were $\leq 0.05$.

\section{Results}

Of the 40 participants in the Sleep study, refer to Table 5, the mean age for the control group was 54.7 years and for the intervention group 56.3 years. Seventy percent of the participants were female in the control group opposed to $65 \%$ female in the intervention group. The analysis of variance (ANOVA) was used to test statistical significance for age by supplement, the $\mathrm{p}$ value was .494 and it is not significant. A Fishers exact test was used to determine statistical significance of race by supplement. The $p$ value was 0.905 and it is not significant. A Fishers exact test was used to determine statistical significance of gender by supplement. The $\mathrm{p}$ value was 1 and it is not significant. No significant differences were identified between the groups related to the demographic characteristics.

The PIRS-20 scale shows sleep improvement when the score is lowered. The control group (see Figure 2) and the slight change in sleep improvement could be attributed as a random result from baseline to week 3. As week 3 to week 6 show no response in either direction. On week 10, when no longer taking the supplement, there was no change. The 5-ALA $50 \mathrm{mg}$ group (see Figure 3), the PIRS-20 score declined from baseline to week 3 and again on week 6 . Week 10, when no longer taking the supplement, the response begins to return to baseline. This is the expected outcome.

Table 4. Sleep study schedule.

\begin{tabular}{|c|c|c|c|c|c|}
\hline Study Schedule & Screening & Intervention Period & Intervention Period & Intervention Period & Follow-Up \\
\hline VISIT SCHEDULE & Screening & $\begin{array}{c}\text { Week 0 } \\
\text { (Visit 1) } \\
\text { Baseline }\end{array}$ & $\begin{array}{r}\text { Week } 3 \\
\text { (Visit 2) }\end{array}$ & $\begin{array}{r}\text { Week } 6 \\
\text { (Visit 3) }\end{array}$ & $\begin{array}{l}\text { Week } 10 \\
\text { (Visit 4) }\end{array}$ \\
\hline Lifestyle Questionnaire \& PIRS-20 Questionnaire & & $\sqrt{ }$ & $\sqrt{ }$ & $\sqrt{ }$ & $\sqrt{ }$ \\
\hline $\begin{array}{c}\text { Clinical Examinations Including Physiological } \\
\text { Measurements }\end{array}$ & $\sqrt{ }$ & $\sqrt{ }$ & $\sqrt{ }$ & $\sqrt{ }$ & $\sqrt{ }$ \\
\hline Laboratory Tests: CBC and Ferritin Level & $\sqrt{ }$ & & & & \\
\hline Intake of Study Supplements & & $\sqrt{ }$ & $\sqrt{ }$ & $\sqrt{ }$ & \\
\hline Filling of Study Diary & & $\sqrt{ }$ & $\sqrt{ }$ & $\sqrt{ }$ & $\sqrt{ }$ \\
\hline Review Participant Concerns & $\sqrt{ }$ & $\sqrt{ }$ & $\sqrt{ }$ & $\sqrt{ }$ & $\sqrt{ }$ \\
\hline
\end{tabular}


Table 5. Baseline statistics.

\begin{tabular}{ccccc}
\hline & \multicolumn{2}{c}{$50 \mathrm{mg} \mathrm{N}=20$} & \multicolumn{2}{c}{ Control N = 20 } \\
\hline & \multicolumn{3}{c}{ Mean Age } & Mean Age \\
\cline { 2 - 5 } Asian/Filipino & $50 \%$ & 57.9 & $45 \%$ & 53.8 \\
Native Hawaiian & $20 \%$ & 53 & $10 \%$ & 62 \\
Caucasian & $25 \%$ & 58.8 & $30 \%$ & 56.16 \\
Hispanic/Latino & $0 \%$ & & $5 \%$ & 43 \\
African American & $5 \%$ & 63 & $5 \%$ & 53 \\
American Indian/ & $0 \%$ & & $5 \%$ & 53 \\
Alaska Native & $0 \%$ & 54.9 & $65 \%$ & 54.61 \\
Female & $70 \%$ & 59.5 & $35 \%$ & 55 \\
Male & $30 \%$ & & &
\end{tabular}

\section{Control} $(\mathrm{N}=\mathbf{2 0})$

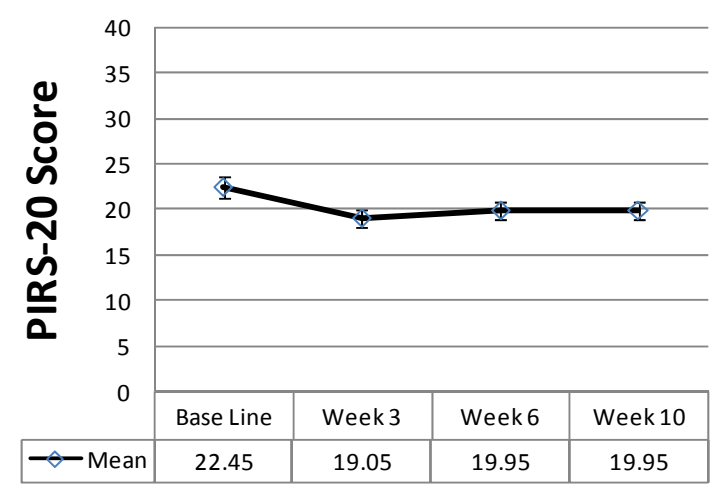

Figure 2. The PIRS-20 scale shows sleep improvement when the score is lowered. The control group and the slight change in sleep improvement could be attributed as a random result from baseline to week 3 . As week 3 to week 6 show no response in either direction. On week 10, when no longer taking the supplement, there was no change.

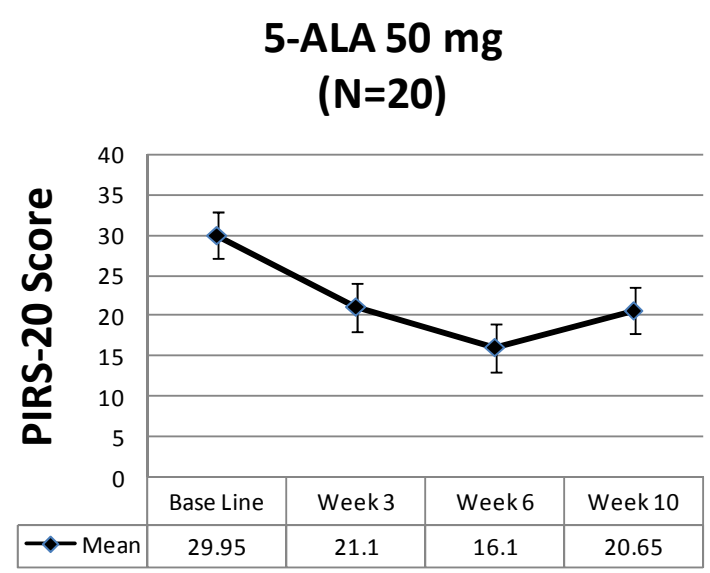

Figure 3. The PIRS-20 score declined from baseline to week 3 and again on week 6 . Week 10, when no longer taking the supplement, the response begins to return to baseline. This is the expected outcome.
Improvement in sleep in the group taking $50 \mathrm{mg} \mathrm{5-}$ ALA, compared to controls, was significant. The mean change, from Baseline through week 6, was -5.67 units less on the sleep scale than the control group with a $p$ value of 0.001 . The mean change from week 6 to week 10 when the participant was no longer taking the supplement was 4.55 units higher than the control with a $p$ value of 0.062 , which is of borderline significance.

In addition, No clinically significant abnormalities were observed in the sleep study or any of the previous studies that could be attributed to the food supplement.

\section{Discussion}

\section{Hypothesis}

Insomnia prevalence in the general population is estimated at $30 \%-50 \%$ [7]. Medications currently used to treat sleep disorders like diphenhydramine, doxylamine and antihistamines have negative side effects. Diphenhydramine side effects include: dry mouth, dizziness, prolonged drowsiness lasting into the next day and memory problems [8]. Doxylamine side effects include: asthma, bronchitis, glaucoma and peptic ulcer or enlarged prostate [9]. Antihistamines can cause dry mouth, urine retention and blurred vision. In addition, all sleep medications cause drowsiness as a solution for treating sleep disorders. Remarkable improvement in sleep was reported by several participants in a previously conducted study investigating the relationship between the dietary supplement 5-ALA and pre-diabetes. The dietary supplement 5-ALA is suggested as being a potential alternative approach to improved sleep. Five-ALA creates energy and may adjust a person's circadian cycle in order to allow for better sleep in a natural way, without negative side effects. For this reason there is a potential interest for natural alternatives for the treatment of sleep disorders.

Increased level of glucose in the cell results in decreased 5-ALA production. Decreased heme production occurs during aging, and at age 40 the human body produces approximately $50 \mathrm{mg}$ /day less [10]. This results in decreased hemoglobin production with decreased heme enzyme activity. A decline in the mitochondrial electron transfer system, the primary cellular energy producer, follows, with decreased basal metabolism, as well as physical energy decline [10].

Melatonin plays a role in regulating circadian rhythms and maintaining physical energy. Melatonin is a chemical that occurs naturally in the brain. Melatonin allows a person to become sleepy. Melatonin is naturally produced during the day-shift phase (see Figure 4).

Endogenous melatonin is produced by the human body. Production begins about two hours before bedtime, provided that the lighting is dim. "This is known as dim- 


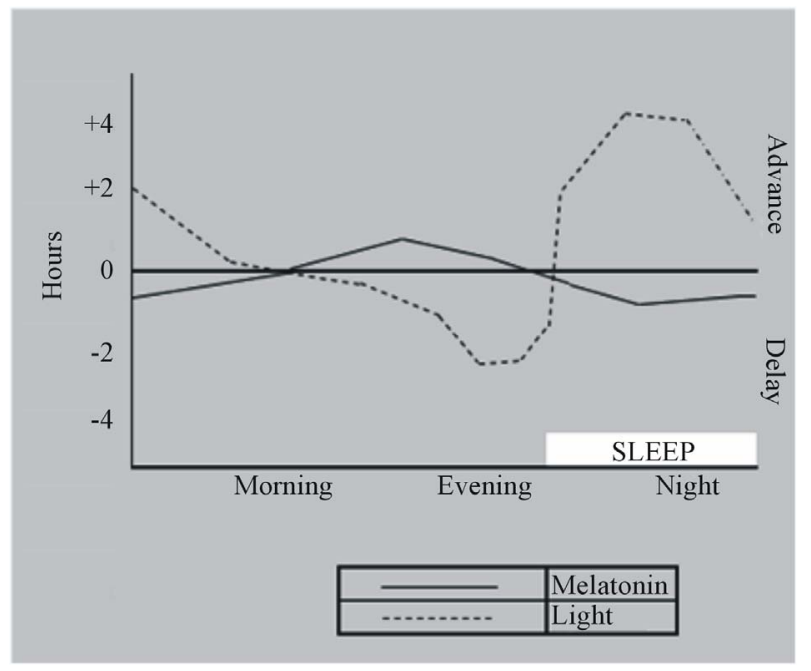

Figure 4. Melatonin plays a role in regulating circadian rhythms and maintaining physical energy. Melatonin is a chemical that occurs naturally in the brain. Melatonin allows a person to become sleepy. Melatonin is naturally produced during the day-shift phase shown in the Phase Response Curve (PRC). Adapted from Lewy et al. and Khalsa et al. $[12,20]$.

light melatonin onset, DLMO" [11]. What results is the phase-advance portion of the phase response curve (PRC) (Figure 4). This assists with regulation of the sleep-wake schedule [12].

According to R. L. Sack, et al., circadian rhythm sleep disorders all involve a problem in the timing of when a person is asleep and is awake [13]. He proposes a master circadian clock in the brain called the suprachiasmatic nucleus (SCN) [14]. The SCN controls the timing of body rhythms related to temperature and hormone levels over a cycle that lasts a little longer than 24 hours.

In order for this system to function efficiently, it needs information from a variety of sources, which include physical activity, social activities and to experience day and night. The ganglion cells in the retina collect light information for the SCN. These cells produce a pigment called melanopsin and are particularly sensitive to light [15]. This is true for "non-image visual functions, such as circadian photo-entrainment and the pupillary light reflex" [15]. The major conduit for rod and cone signals to the brain for non-image visual functions, such as circadian photo-entrainment and the pupillary light reflex [15]. Light exposure is needed by the pineal gland to produce melatonin and the day-phase contributes to melatonin production (see Figure 4) [16]. Melatonin allows a person to be sleepy. "Melatonin is a hormone produced by the pineal gland that contributes to the reinforcement of circadian and seasonal rhythms" [17]. It also helps prepare the body for sleep when melatonin enters phaseadvance and light reduction enters the phase-advance cycle. Extended or enhanced day-phase activity could result in increased melatonin during the evening. 5-ALA could, in fact, enhance day-phase activity, which could inversely allow a person to experience a better sleep cycle.

This is potentially very important as 5-ALA may make for an ideal sleep-aid that assists in inducing drowsiness at night and wakefulness during the day as it minimizes the unwanted side effects related to medications that only cause drowsiness. It should be noted that this particular research was not designed to confirm any change in melatonin levels in the pineal gland. The methods and design did not include testing for melatonin production. Additional studies to evaluate the hypothesis regarding the clinical significance of the use of 5-ALA and a relationship with sleep are needed.

\section{Conclusions and Recommendations}

There appears to be a relationship between the administration of dietary supplements containing 5-ALA and sleep. The results of this study suggest that 5-ALA does, in fact, improve sleep. The mechanism for sleep improvement needs to be explored. Further research is warranted to explore the mechanism by which 5-ALA may play a role in the improvement of sleep.

Current research about 5-ALA and sleep is that 5-ALA has an indirect relationship with intra-cellular energy production and an effect on potentially neuroactive substances such as tryptophan, serotonin, or melatonin. Enhanced cellular energy production could result in a wide range of effects from cellular to endocrine to neurologic. 5-ALA as a key component of heme and the cytochrome system appears to have an impact on increasing the energy of all cells. Research on cytochrome $\mathrm{C}$ oxidase activity and ATP levels in mice in response to 5-ALA appears to confirm this effect [18]. In addition, animal studies indicate a possible effect on neurotransmitters which may have an effect on the sleep-wake cycle.

Many sleep medications induce a tolerance and are recommended only for short-term use. 5-ALA may assist in the adjustment of a person's circadian cycle, endocrine function or neurologic function in order to allow for better sleep in a natural way. In doing so, it may provide for a safer alternative to currently available sleep medication. Further research is needed to explore this possibility.

\section{REFERENCES}

[1] M. Reite, J. Ruddy and K. Nagel, "Concise Guide to Evaluation and Management of Sleep Disorders," 3rd Edition, American Psychiatric Publishing, Inc., Arlington, 2002.

[2] S. Beale, "Biosynthesis of the Tetrapyrrole Pigment Precursor, d-Aminolevulinic Acid, from Glutamate," Plant Physiology, Vol. 93, No. 4, 1990, pp. 1273-1279. 
http://dx.doi.org/10.1104/pp.93.4.1273

[3] M. Doss, F. Sixel-Dietrich and F. Verspohl, "Glucose Effect and Rate Limiting Function of Uroporphyrinogen Synthase on Porphyrin Metabolism in Hepatocyte Culture: Relationship with Human Acute Hepatic Porphyrias," Journal of Clinical Chemistry \& Clinical Biochemistry, Vol. 23, No. 9, 1985, pp. 505-513.

[4] S. Daya, K. O. Nonaka and R. J. Reiter, "Melatonin Counteracts the 5-Aminolevulinic Acidinduced Rise of Rat Forebrain Tryptophan and Serotonin Concentrations at Night," Neuroscience Letters, Vol. 114, No. 1, 1990, pp. 113-116. http://dx.doi.org/10.1016/0304-3940(90)90437-E

[5] D. E. Moul, P. A. Pilkonis, J. M. Miewald, et al., "Preliminary Study of the Test-Retest Reliability and Concurrent Validities of the Pittsburgh Insomnia Rating Scale (PIRS)," Sleep, Vol. 25, Suplement S, 2002, pp. A246A247.

[6] N. Cary, "SAS/STAT ${ }^{\circledR} 9.2$ User's Guide," SAS Institute Inc., Cary, 2008.

[7] S. Carson, M. S. McDonagh, S. Thakurta, P.-Y. Yen and M. Helfand, "Oregon Evidence-Based Practice Center," Oregon Health \& Science University, Portland, 2008.

[8] M. Bayard, T. Avonda and J. Wadzinski, "Restless Legs Syndrome," American Family Physician, Vol. 78, No. 2, 2008, pp. 235-240.

[9] NIH, "Health Information for the Public," National Heart, Lung and Blood Institute, US Department of Health and Human Services, 2009.

[10] B. L. Rodriguez, J. D. Curb, J. Davis, et al., "Use of the Dietary Supplement 5-Aminiolevulinic Acid (5-ALA) and Its Relationship with Glucose Levels and Hemoglobin A1C among Individuals with Prediabetes," Clinical and Translational Science, Vol. 5, No. 4, 2012, pp. 314320. http://dx.doi.org/10.1111/j.1752-8062.2012.00421.x

[11] S. R. Pandi-Perumal, M. Smits, W. Spence, et al., "Dim Light Melatonin Onset (DLMO): A Tool for the Analysis of Circadian Phase in Human Sleep and Chronobiological Disorders," Progress in Neuro-Psychopharmacology \& Biological Psychiatry, Vol. 31, No. 1, 2007, pp. 1-11. http://dx.doi.org/10.1016/j.pnpbp.2006.06.020
[12] A. J. Lewy, V. K. Bauer, S. Ahmed, K. H. Thomas, N. L. Cutler, C. M. Singer, et al., "The Human Phase Response Curve (PRC) to Melatonin is about $12 \mathrm{~h}$ out of Phase with the PRC to Light," Chronobiology International, Vol. 15, No. 1, 1998, pp. 71-83. http://dx.doi.org/10.3109/07420529808998671

[13] R. L. Sack, D. Auckley, R. R. Auger, M. A. Carskadon, K. P. Wright Jr., M. V. Vitiello and I. V. Zhdanova, "An American Academy of Sleep Medicine Revie," Sleep, Vol. 30, No. 11, 2007, pp. 1484-1501.

[14] E. D. Weitzman, C. A. Czeisler, R. M. Coleman, et al., "Delayed Sleep Phase Syndrome. A Chronobiological Disorder with Sleep-Onset Insomnia," Archives of General Psychiatry, Vol. 38, No. 7, 1981, pp. 737-746. http://dx.doi.org/10.1001/archpsyc.1981.0178032001700 $\underline{1}$

[15] M. T. Do and K. W. Yau, "Intrinsically Photosensitive Retinal Ganglion Cells," Physiological Reviews, Vol. 90, No. 4, 2010, pp. 1547-1581. http://dx.doi.org/10.1152/physrev.00013.2010

[16] L. B. Duvall and P. H. Taghert, "Circadian Rhythms: Biological Clocks Work in Phospho-Time," Current Biology, Vol. 21, No. 9, 2011, pp. R305-307. http://dx.doi.org/10.1016/j.cub.2011.04.005

[17] C. M. Morin and R. Benca, "Chronic Insomnia," Lancet, Vol. 379, No. 9821, 2012, pp. 1129-1141. http://dx.doi.org/10.1016/S0140-6736(11)60750-2

[18] S. Ogura, K. Maruyama, Y. Hagiya, Y. Sugiyama, K. Tsuchiya, K. Takahashi, F. Abe, K. Tabata, I. Okura, M. Nakajima, et al., "The Effect of 5-Aminolevulinic Acid on Cytochrome Coxidase Activity in Mouse Liver," BMC Research Notes, Vol. 4, No. 1, 2011, p. 66. http://dx.doi.org/10.1186/1756-0500-4-66

[19] CSID:134, 2013. http://www.chemspider.com/Chemical-Structure.134.html

[20] S. B. Khalsa, M. E. Jewett, C. Cajochen, et al., "A Phase Response Curve to Single Bright Light Pulses in Human Subjects," The Journal of Physiology, Vol. 549, No. 3, 2003, pp. 945-952.

http://dx.doi.org/10.1113/jphysiol.2003.040477 